\title{
Insight into physical activity in combating the infantile metabolic syndrome
}

\author{
Benjamin C. Guinhouya · Hervé Hubert
}

Received: 27 May 2010/Accepted: 29 September 2010/Published online: 26 October 2010

(c) The Japanese Society for Hygiene 2010

\begin{abstract}
Metabolic syndrome (MetS) is increasingly reported in children, mainly in the presence of overweight/ obesity. From the most recent report, up to $60 \%$ of overweight and obese children can be affected by this syndrome. MetS acquired during childhood has been shown to track into adulthood, including its clinical complications, such as type 2 diabetes and cardiovascular diseases. Among the practical preventive and therapeutic measures to be taken in children, physical activity (PA) appears to be at least as efficient as the most adequate pharmacology. The current literature suggests that exercise programs based either on aerobic-or resistance-type exercises, or a combination of these 2 types of structured activity, may promote insulin sensitivity and weaken or suppress MetS in children. Furthermore, daily-living activities such as brisk walking were found to substantially reduce the risk of MetS among children. Regardless of their weight status, PA needs to be promoted among children as early as possible.
\end{abstract}

Keywords Exercise · Health · Insulin · Lifestyle · Obesity

\section{Introduction}

Metabolic syndrome (MetS) is regarded as the clustering of at least three risk factors that fit the following criteria: hypertriglyceridemia, hypertension, abdominal obesity,

B. C. Guinhouya $(\square) \cdot$ H. Hubert

Laboratory of Public Health: EA 2694,

Faculty of Engineering and Management in Health,

UDSL/ILIS, Univ. Lille Northern France,

42, rue Ambroise Paré, 59120 Loos, France

e-mail: benjamin.guinhouya@univ-lille2.fr low concentration of high-density lipoprotein (HDL)-cholesterol (HDL-C), and high fasting blood glucose. Overt MetS during childhood is indicative of an increased risk of morbidity-mortality from type 2 diabetes and cardiovascular diseases (CVDs), and a loss of functional capacities throughout life. This observation highlights the magnitude of the future health and economic consequences at the individual and society levels.

Various definitions have been used for infantile MetS, including adult-adapted standards such as the National Cholesterol Education Program-Adult Treatment Panel III (NCEP-ATP III) [1], the International Diabetes Federation (IDF) criteria [2], and the Japanese criteria [3]. Some authors have even suggested using a continuous measure for MetS in children rather than a dichotomous assessment [4-6], and a study from Japan has pinpointed glutamate pyruvate transaminase as a better marker of MetS in children than fasting blood glucose [7]. Thus the definition of the MetS for the pediatric population remains a matter of debate. Nevertheless, whatever the diagnostic criteria, compelling data have revealed that the prevalence of pediatric MetS is high and increases with worsening obesity in obese children. For instance, Saland [8] has found that the prevalence of MetS ranges from 18 to $50 \%$ in obese children, while it occurs in $<1 \%$ of normal-weight children. More recently, it has been reported that up $60 \%$ of overweight and obese children may be affected [9]. Because obesity is intimately associated with pediatric MetS [10], which may track into adulthood, including its clinical complications [11, 12], the high proportion of overweight/obese children observed over the past few years should be considered symptomatic of a larger decline in the overall health of the population. In the United States, and Europe as well, the number of overweight or obese children has doubled, and the number of obese adolescents has tripled over the past 20 years [13]. As an 
indication of this trend, the prevalence of overweight/obese youth increased from 3 to $>15 \%$ in 40 years in France, for example. Stated simply, if, over the next 40 years, lifestyles remain identical to those in the past 40 years, then in 2040, $>50 \%$ of French adults will have MetS and its associated clinical conditions. The best strategies to prevent and/or treat infantile MetS must be determined. From this perspective, physical activity (PA) appears to be one of the most effective preventive and therapeutic options, and a number of physiological explanations have been provided regarding the mechanisms through which PA may positively impact MetS or its components [14-16]. Thus, it has been suggested that PA can directly influence insulin resistance while causing, inter alia, the short-term activation of glucose transporter (GLUT-4) receptors. This occurs through lowering the circulating insulin level by increasing the production of anti-inflammatory factors, such as adiponectin, or by inducing an increase in the quantity of oxidative and insulin-sensitive muscle fibers. These effects can be direct or they can be mediated by an increase in physical fitness [cardiorespiratory fitness (CRF) and strength] or by a decrease of adiposity (which may occur in direct correlation with an increased circulating concentration of adiponectin). Further research work may elucidate these mechanisms. Meanwhile, a growing number of studies lend support to a negative relationship between different forms of PA and infantile MetS or its components.

Physical activity is a complex behavior that involves the movements of skeletal muscles. In theory, it includes all bodily movements from fidgeting to sports, but it should not be limited to any of its forms. Exercise is a subtype of PA and can be grouped into flexibility, aerobic, and anaerobic/ resistance exercises, according to their overall effects on the body. Aerobic and resistance exercises appear to play a significant role in protecting children against MetS.

Although it is believed that a combination of dietary and PA interventions may be the most beneficial for reducing the risk of MetS, recent data suggest that PA alone could attenuate or remove infantile MetS or its components through its insulin-sensitizing affects on skeletal muscle. As shown in Fig. 1, the main target of PA and exercise programs is insulin resistance [17], although regular PA can also counteract the syndrome's various biological risk factors, such as high blood pressure and hyperlipidemia. These effects of PA can be obtained through structured exercise programs and by daily-living PA as well.

\section{Influence of structured exercise programs: aerobic and resistance training}

It has been found that aerobic exercise and well-supervised resistance training among children have positive

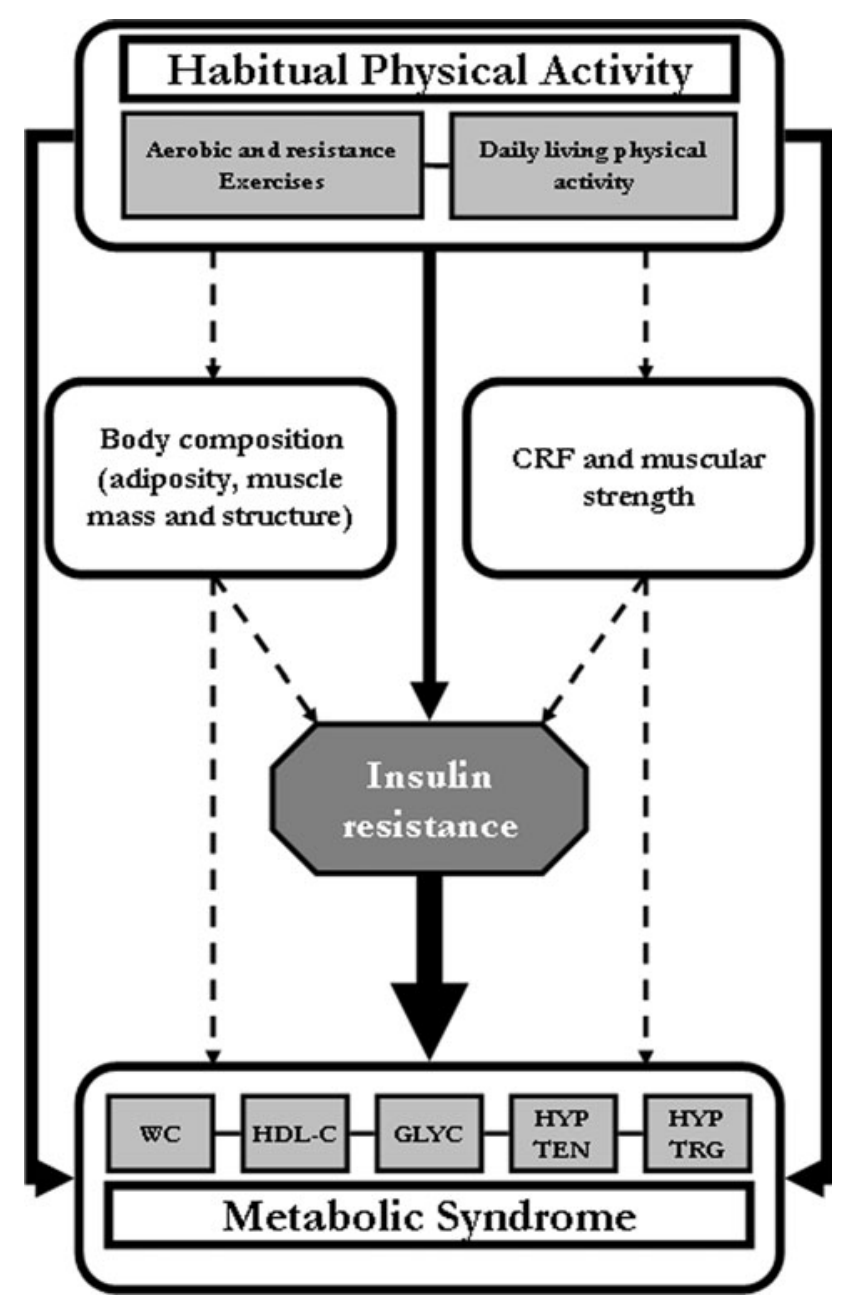

Fig. 1 Simplified diagram of the action of physical activity on insulin resistance and the metabolic syndrome. Evidenced direct and indirect effects of physical activity on insulin resistance and on the different components of the metabolic syndrome are indicated by solid lines. Effects of physical activity through the joint improvement of physical fitness and body composition are indicated by dashed lines. CRF Cardiorespiratory fitness, WC waist circumference, $H D L-C$ highdensity lipoprotein-cholesterol, GLYC high fasting blood glucose, HYPTEN hypertension, HYPTRG hypertriglyceridemia

effects on insulin resistance and various components of infantile MetS. Nassis et al. [18] found an improvement of insulin sensitivity in obese children after they had participated in a 12-week exercise training program. The concentration of circulating hormones was not modified, but the program produced a $12 \%$ increase in CRF. Increased CRF, achievable through aerobic exercise, may protect against infantile MetS even in the presence of excess weight. Likewise, some resistance exercises have been found to increase muscular strength and protect against overly high insulin resistance, regardless of an increase in CRF [19]. Finally, combining aerobic and resistance exercises could be used to improve insulin sensitivity in children [20,21]. 


\section{Influence of daily-living physical activity}

Apart from the positive effects (acute or medium-term) of structured exercises on insulin sensitivity and MetS, some cross-sectional and longitudinal data illustrate the significance of habitual physical activity (HPA) in preventing infantile MetS. Consistent negative relationships have been found between insulin resistance, MetS or its indicators, and HPA in children, measured using a questionnaire or objectively through accelerometry. Commonly, this relationship is independent of other factors (e.g., body composition, physical fitness) [22, 23]. Nonetheless, there may be a longitudinal joint increase in central adiposity and insulin resistance in children when PA is decreased [15]. Moreover, it has been suggested that the beneficial effects of PA are better in children whose CRF is initially weak [22]. Mechanisms similar to those mentioned above may explain these variations. However, it is important to acknowledge that these positive effects are evident even if the intensity of daily-living activities sufficiently stimulates the skeletal muscles or adipose tissue, producing appropriate physiological adaptations likely to improve insulin activity. Some recent data suggest that 30-40 min of brisk walking (or an equivalent activity) per day can reduce MetS risk by one-third [24]. Consequently, it is an overwhelming public health challenge to help children make optimal use of their available opportunities for activity to counter obesity and its comorbidities.

At an individual level, children can engage in PA through structured activities (e.g., physical education lessons or sports) and discretionary activities (e.g., recess activities). While the role of teachers and the school environment should not be neglected, parental support is required to reinforce the value of PA among children. Health professionals, and especially general practitioners and pediatricians, need to learn about and include PA counseling in their practice [25] so as to help children and their families in incorporating an active lifestyle in their routine. Finally, it is the role of policy-makers and the entire community to create a safe and fun environment for children to enjoy PA outdoors.

\section{References}

1. Dubose KD, Stewart EE, Charbonneau SR, Mayo MS, Donnelly JE. Prevalence of the metabolic syndrome in elementary school children. Acta Paediatr. 2006;95:1005-1011.

2. Zimmet P, Alberti G, Kaufman F, et al. The metabolic syndrome in children and adolescents-an IDF consensus report. Pediatr Diabetes. 2007;8:299-306.

3. Ozeki T, Nakagawa Y, Nakanishi $T$, et al. Discussion about definition criteria of the metabolic syndrome in children: the general research for preventing lifestyle diseases such as cardiovascular disease, "Cohort study for establishment of concepts, pathology and definition criteria, and effective medical intervention of the metabolic syndrome in children". Labour and Welfare Ministry of Japan, Annual reports of Health. 2006; $5-8$.

4. Eisenmann JC. On the use of a continuous metabolic syndrome score in pediatric research. Cardiovasc Diabetol. 2008;7:1-6.

5. Eisenmann JC, Laurson KR, DuBose KD, Smith BK, Donnelly JE. Construct validity of a continuous metabolic syndrome score in children. Diabetol Metab Syndr. 2010;2:1-8.

6. Thivel D, Malina RM, Isacco L, et al. Metabolic syndrome in obese children and adolescents: dichotomous or continuous? Metab Syndr Relat Disord. 2009;7:549-55.

7. Abe Y, Kikuchi T, Nagasaki K, et al. Usefulness of GPT for diagnosis of metabolic syndrome in obese Japanese children. J Atheroscler Thromb. 2009;16:902-9.

8. Saland JM. Update on the metabolic syndrome in children. Curr Opin Pediatr. 2008;19:183-91.

9. Tailor AM, Peeters PH, Norat T, Vineis P, Romaguera D. An update on the prevalence of the metabolic syndrome in children and adolescents. Int J Pediatr Obes. 2010;5:202-13.

10. Weiss R, Dziura J, Burgert TS, Tamborlane WV, Taksali SE, et al. Obesity and the metabolic syndrome in children and adolescents. N Engl J Med. 2004;350:2362-74.

11. Camhi S, Katzmarzyk PT. Tracking of cardiometabolic risk factor clustering from childhood to adulthood. Int J Pediatr Obes. 2009;10:1-8.

12. Morrison JA, Friedman LA, Wang P, Glueck CJ. Metabolic syndrome in childhood predicts adult metabolic syndrome and type 2 diabetes mellitus 25 to 30 years later. J Pediatr. 2008;152:201-6.

13. MacPhee M. Global childhood obesity: how to curb an epidemic. J Pediatr Nurs. 2008;23:1-4.

14. Houmard JA, Egan PC, Neufer PD, et al. Elevated skeletal muscle glucose transporter levels in exercise-trained middle aged men. Am J Physiol. 1991;261:E437-43.

15. Jago R, Wedderkopp N, Kristensen PL, et al. Six-year change in youth physical activity and effect on fasting insulin and HOMAIR. Am J Prev Med. 2008;35:554-60.

16. Roberts CK, Barnard RJ, Scheck SH. Exercise-stimulated glucose transport in skeletal muscle is nitric oxide dependent. Am J Physiol. 1997;273:E220-5.

17. Guinhouya BC. Rôle de l'activité physique dans la lutte contre le syndrome métabolique infantile. Med Sci (Paris). 2009;25: 827-34.

18. Nassis GP, Papantakou K, Skenderi K, Triandafillopoulou M, et al. Aerobic exercise training improves insulin sensitivity without changes in body weight, body fat, adiponectin, and inflammatory markers in overweight and obese girls. Metabolism. 2005;54:1472-9.

19. Benson AC, Torode M, Fiatarone Singh MA. Muscular strength and cardiorespiratory fitness is associated with higher insulin sensitivity in children and adolescents. Int $\mathrm{J}$ Pediatr Obes. 2006;1:222-31.

20. Ahmadizad S, Haghighi AH, Hamedinia MR. Effects of resistance versus endurance training on serum adiponectin and insulin resistance index. Eur J Endocrinol. 2007;157:625-31.

21. Bell LM, Watts K, Siafariskas A, et al. Exercise alone reduces insulin resistance in obese children independently of changes in body composition. J Clin Endocrinol Metab. 2007;92:4230-5.

22. Brage S, Wedderkopp N, Ekelund U, et al. Features of the metabolic syndrome are associated with objectively measured physical activity and fitness in children: the European Youth Heart Study (EYHS). Diabetes Care. 2004;27:2141-8.

23. Kelishadi R, Razaghi EM, Gouya MM, Ardalan G, et al. Association of physical activity and the metabolic syndrome in 
children and adolescents: CASPIAN study. Horm Res. 2007;67: 46-52.

24. Ekelund U, Anderssen S, Andersen L-B, Riddoch C, et al. Prevalence and correlates of the metabolic syndrome in a population-based sample of European youth. Am J Clin Nutr. 2009;89:90-6.

25. Estabrooks PA, Glasgow RE, Dzewaltowski DA. Physical activity promotion through primary care. JAMA. 2003;289:2913-6. 